\title{
Three-dimensional acetylenic modified graphene for high- performance optoelectronics and topological materials
}

\author{
Yan Gao $\mathbb{i D}^{1,5}$, Chengyong Zhong $\mathbb{1 D}^{2,5}$, Shengyuan A. Yang $\mathbb{( D}^{3,4}$, Kai Liu (iD) ${ }^{1 凶}$ and Zhong-Yi Lu (iD)
}

Seeking carbon phases with versatile properties is one of the fundamental goals in physics, chemistry, and materials science. Here, based on the first-principles calculations, a family of three-dimensional (3D) graphene networks with abundant and fabulous electronic properties, including rarely reported dipole-allowed truly direct band gap semiconductors with suitable band gaps (1.07-1.87 eV) as optoelectronic/photovoltaic materials and topological nodal-ring semimetals, are proposed through stitching different graphene layers with acetylenic linkages. Remarkably, the optical absorption coefficients in some of those semiconducting carbon allotropes express possibly the highest performance among all of the semiconducting carbon phases known to date. On the other hand, the topological states in those topological nodal-ring semimetals are protected by the time-reversal and spatial symmetry and present nodal rings and nodal helical loops topological patterns. Those newly revealed carbon phases possess low formation energies and excellent thermodynamic stabilities; thus, they not only host a great potential in the application of optoelectronics, photovoltaics, and quantum topological materials etc., but also can be utilized as catalysis, molecule sieves or Liion anode materials and so on. Moreover, the approach used here to design novel carbon allotropes may also give more enlightenments to create various carbon phases with different applications.

npj Computational Materials (2021)7:109; https://doi.org/10.1038/s41524-021-00579-5

\section{INTRODUCTION}

Carbon is one of the central and essential elements in the current scientific research and industrial applications due to its novel properties, i.e., superhardness, exceptional toughness, and the highest thermal conductivity ${ }^{1-7}$. Although elemental carbon exists in two natural allotropes, i.e., diamond and graphite, there are also many novel carbon phases that have been manufactured in experiments over the past decades, such as fullerenes, carbon nanotubes, graphene, and so on ${ }^{8,9}$. Of them, graphene has a very central and vital role in that fullerenes can be viewed as wrappedup graphene and carbon nanotubes can also be made of graphene by rolling up along specific direction. Moreover, lots of methods have been proposed to obtain novel carbon allotropes based on graphene. For instance, a new family of two-dimensional (2D) carbon materials, graphynes, has been revealed by replacing different single bonds in graphene with acetylenic bonds ${ }^{10}$. Using graphene nanoribbons as building blocks, a series of 3D graphene networks have been obtained and intensively studied in various fields ${ }^{11-14}$. Therefore, it occurs to us whether or not there is another possibility to design novel carbon polymorphs from graphene.

It should be emphasized that we are not just curious about how many esthetic carbon phases there are, more importantly, looking back in the last decades, the discovery and invention of new forms of carbon have always opened doors to new science and technology ${ }^{15-18}$. Such as the fullerenes, carbon nanotubes and graphene have been immensely studied from catalysis, energy storage, optoelectronics, photovoltaics, organic electronics, etc. ${ }^{19,20}$. One of the most important topics in developing carbon materials is to replace silicon-based electronics with carbon-based electronics ${ }^{21}$, which need carbon phases with appropriate direct band gap and well electron transition of optical absorption. It is well-known that semiconductors are classified into direct and indirect band gap semiconductors, respectively, depending on whether their valence band maximum (VBM) and conduction band minimum (CBM) occur at the same wavevector in reciprocal space. However, only when the optical transition selection rules are satisfied by the symmetries of wavefunctions of VBM, CBM, and transition dipole operator, the semiconductors can be viewed as dipole-allowed truly direct band gap semiconductors ${ }^{22}$, which are really pursued by us. Unfortunately, diamond is unsuitable due to its wide indirect band gap $(\sim 5.5 \mathrm{eV})$. Despite many efforts being paid to search high-performance semiconducting carbon allotropes, such as recent experimentally synthesized T-Carbon ${ }^{23,24}$ or theoretically proposed $\mathrm{CKL}^{25,26}$ and $\mathrm{CYKL}^{27}$, etc., they are not dipole-allowed truly direct band gap semiconductors and may encounter challenges in chemical synthesis because of their high total energies or structural complexities. Thus, it is highly desired to seek a high-performance semiconducting carbon phase with great potential of being realized.

On the other hand, studying physics in carbon materials also created a new era in condensed matter physics, i.e., topological physics in solid states ${ }^{28-30}$, as evidenced by the Dirac fermion and quantum spin Hall effect in graphene ${ }^{31}$. Although topological insulator was first proposed in graphene, its negligible spinorbital coupling (SOC) is not suitable for inducing observable topological effects at ambient condition. Therefore, the study of a topological insulator is now mainly concentrated on heavy element compounds with strong SOC that can induce more prominent topological effects. However, carbon, as a light element, is more preferable for spawning topological semimetal

\footnotetext{
${ }^{1}$ Department of physics and Beijing Key Laboratory of Opto-electronic Functional Materials \& Micro-nano Devices, Renmin University of China, Beijing, China. ${ }^{2}$ Institute for Advanced Study, Chengdu University, Chengdu, China. ${ }^{3}$ Research Laboratory for Quantum Materials, Singapore University of Technology and Design, Singapore, Singapore. ${ }^{4}$ Center for Quantum Transport and Thermal Energy Science, School of Physics and Technology, Nanjing Normal University, Nanjing, China. ${ }^{5}$ These authors contributed equally: Yan Gao, Chengyong Zhong. ${ }^{凶}$ email: kliu@ruc.edu.cn; zlu@ruc.edu.cn
} 
since the SOC is not mandatory in topological semimetal ${ }^{32-36}$. In fact, there are lots of topological semimetal states, such as topological nodal line semimetal ${ }^{37-39}$, topological nodal surface semimetal $^{40-42}$, or new topological fermions ${ }^{43,44}$, have been proposed in carbon materials theoretically. Unfortunately, we notice that none of those reported topological semimetals in carbon materials have been realized in experiments, for which we think the reason is partial that those proposed carbon structures have relatively high total energies and lack any available structural precursors that can be used to realize the proposed carbon structures. Thus, searching for topological semimetal in carbon materials from the perspective of easy synthesis is still challenging and desirable.

In this work, we propose a new strategy to tailor graphene through stitching graphene layers with acetylenic linkage, as shown in Fig. 1a, b, which here is termed as 3D acetylenic modified graphene (3D-AMG). According to our first-principles calculations, all of the 3D-AMGs demonstrate excellent stabilities, as confirmed by their total energies, phonon spectra, and ab initio molecular dynamics (AIMD). Prominently, the family of 3D-AMGs present very abundant and fabulous electronic properties, some of which are dipole-allowed truly direct band gap semiconductors with band gaps in the range of 1.0-1.5 eV, which are located in the optimal band gap value range for photovoltaic application, and possess probably the highest optical absorption strength amongst all of the semiconducting carbon allotropes known to date, especially the band gap of 3D-AMG-7 is $1.19 \mathrm{eV}$ that is very close to the optimal value of $1.34 \mathrm{eV}$ for solar cell material; on the other hand, some 3D-AMGs are topological nodal-ring semimetals. Our work discovers some semiconducting carbon allotropes with excellent optical absorption strength and some topological nodalring semimetals, meanwhile the approach used here to design novel carbon allotropes through connecting graphene with acetylenic linkage may also give more thoughts to create various carbon phases with different applications.

\section{RESULTS}

The structure information and the stabilities of 3D-AMGs

As shown in Fig. $1 \mathrm{a}, \mathrm{b}$, the graphene layers $\left(s p^{2}\right.$-hybridized atoms colored with blue and denoted as (2) are stitched vertically by acetylenic linkage ( $s p$-hybridized atoms colored with red and denoted as $\mathrm{C1}$ ) going through a carbon dimer $\left(s p^{3}\right.$-hybridized atoms colored with cyan and denoted as (3) along the armchair direction of graphene, so the family of 3D-AMG- $n$ is obtained, where $n$ denotes the width of the armchair nanoribbons in between two acetylenic connected carbon dimer (i.e., the cyan atoms). Thus Fig. 1a, b are 3D-AMG-4 and 3D-AMG-5, respectively. There are two space groups in 3D-AMG- $n$ depending on $n$ being odd $(\mathrm{P} 2 / \mathrm{M})$ or even $(\mathrm{C} 2 / \mathrm{M})$. As two representatives, we tabulate the basic structural information of 3D-AMG-4 and 3D-AMG-5 in Table 1 together with other carbon allotropes such as the CKL, CYKL, diamond, graphite, and T-carbon for comparison, and those of the rest members of 3D-AMG- $n$ can be found in supplementary Table 1.

One would expect 3D-AMGs should express good stability since they are constructed from graphene. It is actually true. The calculated total energies of 3D-AMG-4 and 3D-AMG-5 are $-8.86 \mathrm{eV}$ and -8.91 per atom, respectively, which are $\sim 0.05 \sim 1.00 \mathrm{eV} /$ atom lower than those of the previously reported CKL ( $-8.81 \mathrm{eV} /$ atom), CYKL ( $-8.24 \mathrm{eV} /$ atom), and recently realized T-carbon ( $-7.91 \mathrm{eV} /$ atom). Moreover, the total energies decrease as the increase of $n$ in 3D-AMG- $n$ (see supplementary Table 1), such as the total energy of 3D-AMG-10 is $-9.03 \mathrm{eV} /$ atom, which almost approaches that of diamond ( $-9.09 \mathrm{eV} /$ atom). On the other hand, the phonon spectra of 3D-AMG-4 and 3D-AMG-5 have no soft mode throughout the entire Brillion zone (BZ), implying they are also dynamically stable (see Fig. 2a, b). Their AIMD simulations manifest that they can be stable under at least $1000 \mathrm{~K}$ (see Fig. $2 c-f)$, showing very excellent thermodynamic stability as well.

\section{The electronic and optical properties of 3D-AMGs}

Now, we proceed to investigate the electronic properties of 3DAMGs. It has been known that the main drawback of graphene to

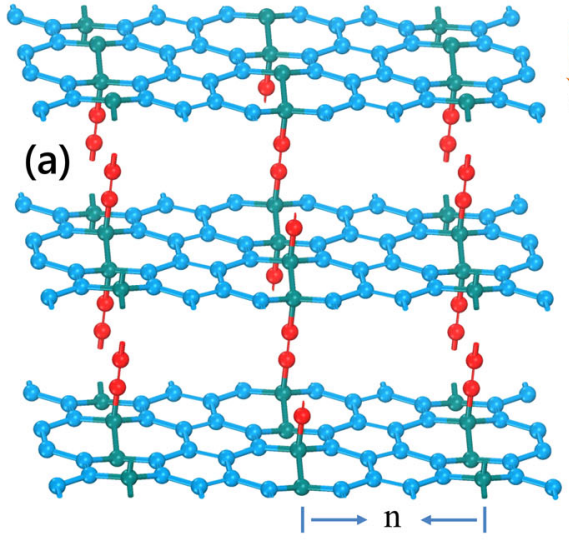

(c)

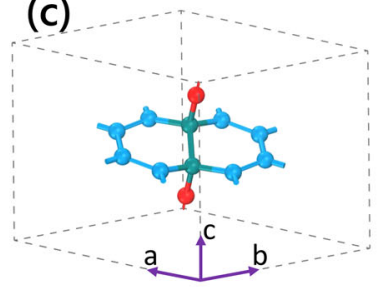

(d)

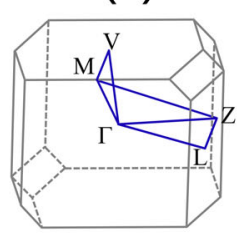

(b)

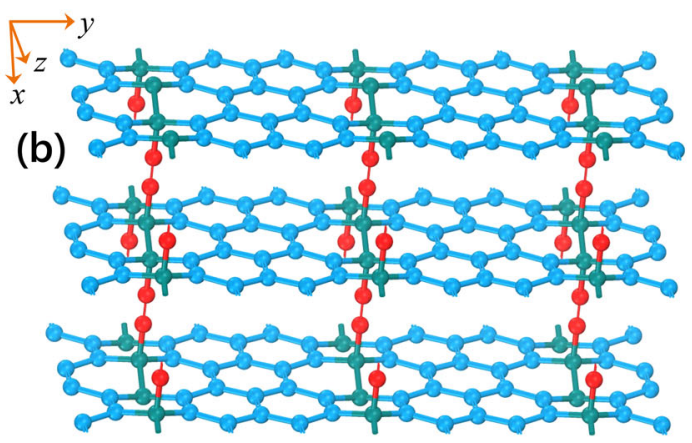

$\mathrm{C} 1(s p) \bigcirc \mathrm{C} 2\left(s p^{2}\right) \bigcirc \mathrm{C} 3\left(s p^{3}\right)$

(e)

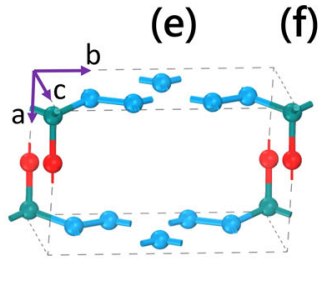

(001)

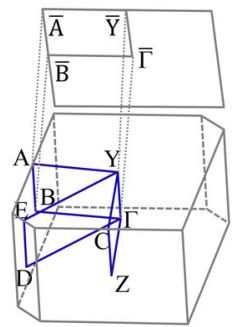

Fig. 1 Crystal structure and Brillion zone (BZ) of 3D-AMG-n. The optimized atomic structure of a 3D-AMG-4 and b 3D-AMG-5. The $s p$-, $s p^{2}-$, and $s p^{3}$-hybrized carbon atoms are colored (denoted) with red (C1), blue (C2) and cyan (C3), respectively. c Primitive cell and d the BZ of 3DAMG-4. e Primitive cell and $\mathbf{f}$ the BZ with the projected 2D BZ of the (001) surface of 3D-AMG-5. 
Table 1. Crystal system, space group (SG), lattice parameters a/b/c $(\AA)$ and $\alpha / \beta / \gamma\left({ }^{\circ}\right)$, mass density $\rho\left(\mathrm{g} / \mathrm{cm}^{3}\right)$, band gap $E_{\mathrm{g}}(\mathrm{eV})$, and total energy $E_{\mathrm{t}}(\mathrm{eV} /$ atom) of 3D-AMG-4, 3D-AMG-5, diamond, CKL, CYKL, and T-carbon obtained from our work in comparison with those in literature.

\begin{tabular}{|c|c|c|c|c|c|c|c|c|c|c|c|}
\hline System & SG & Method & \multicolumn{6}{|c|}{ Lattice parameters } & $\rho$ & $E_{\mathrm{g}}$ & $E_{\mathrm{t}}$ \\
\hline 3D-AMG-4 & $\mathrm{C} 2 / \mathrm{M}$ & Our work & 6.59 & 6.59 & 6.06 & 101.21 & 101.21 & 142.17 & 1.85 & $1.31(1.87)$ & -8.86 \\
\hline 3D-AMG-5 & $\mathrm{P} 2 / \mathrm{M}$ & Our work & 4.89 & 7.47 & 4.28 & 90.00 & 98.09 & 90.00 & 1.81 & Semimetal & -8.91 \\
\hline \multirow[t]{2}{*}{ Diamond } & $\mathrm{FD} \overline{3} \mathrm{M}$ & Our work & 2.53 & & & & & & 3.55 & 4.12 & -9.09 \\
\hline & & $\mathrm{GGA}^{66}$ & 2.53 & & & & & & 3.50 & $(5.34)$ & -9.09 \\
\hline CKL & & $\mathrm{GGA}^{26}$ & 4.46 & & 2.53 & & & & 2.75 & $2.35(3.43)$ & -8.81 \\
\hline \multirow[t]{2}{*}{ CYKL } & $\mathrm{F}_{3} / \mathrm{MMC}$ & Our work & 6.99 & & 6.85 & & & & 1.24 & $1.81(2.71)$ & -8.24 \\
\hline & & $\mathrm{GGA}^{27}$ & 6.99 & & 6.85 & & & & 1.23 & $1.81(2.70)$ & -8.24 \\
\hline \multirow[t]{2}{*}{ T-Carbon } & $\mathrm{FD} \overline{3} \mathrm{M}$ & Our work & 7.52 & & & & & & 1.50 & $1.47(2.18)$ & -7.91 \\
\hline & & $\mathrm{GGA}^{68}$ & 7.52 & & & & & & 1.50 & $(2.27)$ & -7.92 \\
\hline
\end{tabular}

The values of band gap in the parentheses are the results calculated with the HSEO6 functional.

impede its application in semiconductor devices is the absence of band gap. Focusing on the band gap's engineering of graphene, there are plenty of methods that have been proposed, such as cutting into graphene nanoribbons or surface functionalization ${ }^{45}$. The acetylenic modification here on the surface of graphene can be treated as the extension of the method of surface functionalization, but just of extending to three dimension by connecting different graphene layer with a functional group (i.e., the acetylenic linkage). Based on our first-principles calculations, these 3D-AMGs demonstrate very abundant and fascinating electronic properties. Altering the value of $n$ in 3D-AMGs, indirect band gap, direct band gap semiconductors, and topological nodal-ring semimetals can be found. Here, we are mainly interested in the direct band gap semiconductors and topological nodal-ring semimetals, thus they are discussed elaborately below as exemplified with 3D-AMG-4 and 3D-AMG-5, respectively. The results of the rest members of 3D-AMGs can be found in Supplementary Information (SI).

The band structure of 3D-AMG-4 is shown in Fig. 3a. One can clearly observe that the VBM and the CBM locate at the same M point, indicating that it is a direct band gap semiconductor. Although 3D-AMG-4 is a direct band gap semiconductor, it is known that in a crystal with inversion symmetry there are likely parity-forbidden transitions induced between conduction and valence band edges, which can in turn seriously affect its optical absorption properties ${ }^{46}$. The space group of $3 \mathrm{D}-\mathrm{AMG}-4$ is $\mathrm{C} 2 / \mathrm{M}$ containing the inversion symmetry, therefore more information is needed to analyze its optical performance. According to our firstprinciples calculations, the VBM and CBM of 3D-AMG-4 are mainly derived from the $p$ orbitals of C2 carbon atoms (see Fig. 3b) and have respective odd and even parities (denoting with "-" and "+" in Fig. 3a), which means 3D-AMG-4 is a direct band gap semiconductor exhibiting no parity-forbidden (even to even or odd to odd) transitions.

The transition probabilities between the topmost valence and the lowest conduction bands can be revealed by the sum of the squares of the dipole transition matrix elements (TME), $P^{2}$, at various $k$ points ${ }^{46}$. The calculated TME of 3D-AMG-4 is shown in Fig. 3c. One can observe that the high transition probability at $M$ point, implying its potential strong optical absorption strength. Indeed, the calculated optical properties of 3D-AMG-4, namely, the imaginary part of dielectric function $\varepsilon_{2}(E)$ demonstrates very strong strength in comparison with the previously reported dipole-allowed truly direct band gap semiconductor CYKL/CKL and semiconducting T-carbon/diamond. Moreover, the other members of 3D-AMG- $n$ with direct band gaps exhibit the same features (see supplementary Fig. 5). Small effective masses of 3DAMG-4 are expected in consideration of its large band dispersions near the VBM and CBM. The electron and hole effective masses of 3D-AMG-4 are tabulated in Table 2 along with those of other direct band gap semiconducting carbon allotropes-CKL, CYKL, and T-Carbon. One can observe that 3D-AMG-4 shows very small effective masses in the graphene plane (i.e., $m_{e}^{\|}$and $m_{h h}^{\|}$). Furthermore, the PBE (HSE06) band gaps of 3D-AMG-3/4/7/10 are $0.62 \mathrm{eV}(1.12 \mathrm{eV}), 1.31 \mathrm{eV}(1.87 \mathrm{eV}), 0.82 \mathrm{eV}(1.19 \mathrm{eV})$, and $0.71 \mathrm{eV}(1.07 \mathrm{eV})$, respectively (see Tab. 2, supplementary Fig. 6 and supplementary Fig. 7), which make them quite suitable for solar cell materials, according to the Shockley-Queisser's theory ${ }^{47}$ that the optimal band gap for solar cell materials should straddle between 1.0 and $1.5 \mathrm{eV}$.

\section{The topological properties of 3D-AMGs}

Another interesting feature of 3D-AMG- $n$ family is that some of them are topological nodal-ring semimetals as exemplified by 3DAMG-5 here. The band structure of 3D-AMG-5 is shown Fig. $4 a$, in which the Dirac point crossed by the valence and conduction bands along B-A can be clearly observed. We also confirm the results by the HSE calculation (see supplementary Fig. 6b). According to our calculations, the bands near the Fermi level are mainly contributed from the $p_{x}$ orbitals of C2 atoms, as presented in the charge densities and PDOS (see the inset of Fig. $4 a, b)$. Based on those analysis, we develop a tight-binding (TB) model to describe the hopping integrals in 3D-AMG-5, and the TB band structure fits very well with the density functional theory (DFT) results around the Fermi level (more details can be found in $\mathrm{SI})$.

3D-AMG-5 has the little point group of $C_{2}$ along the B-A path, and the two bands involved in the crossing belong to different irreducible representations of A and B (see the inset in Fig. 4a), implying that the crossed point is protected by crystalline symmetry. In addition, the system preserves the inversion $(P)$ and time-reversal $(T)$ symmetries. For such $P T$-symmetric system without SOC, the crossed point cannot be an isolated single point. Indeed, a careful inspection reveals that the band crossing points form a continuous nodal ring in the whole BZ (see the inset in Fig. 4a). This nodal ring is protected by a quantized one-dimensional 

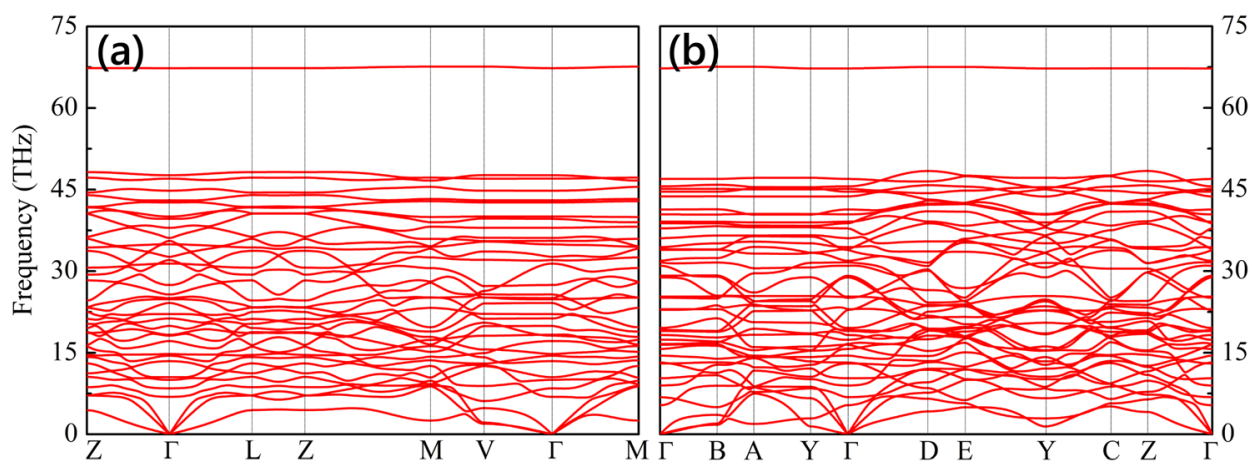

(c) $1000 \mathrm{~K}$
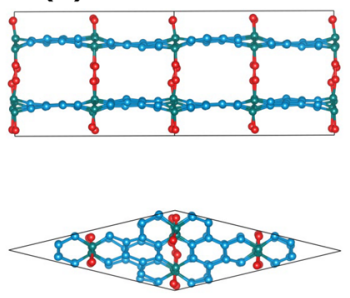

(d) $1200 \mathrm{~K}$
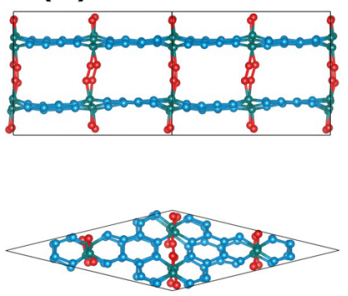

(e) $1000 \mathrm{~K}$
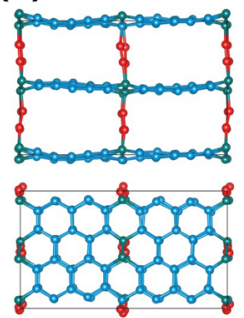

(f) $1200 \mathrm{~K}$
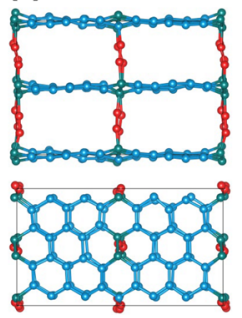

Fig. 2 Dynamical and thermal stabilities. Phonon dispersions of a 3D-AMG-4 and $\mathbf{b}$ 3D-AMG-5 in the entire BZ. c-f The side (top panel) and top (bottom panel) views of the snapshots of 3D-AMG-4 and 3D-AMG-5 at the temperatures of $1000 \mathrm{~K}$ and $1200 \mathrm{~K}$ after $15-\mathrm{ps}$ AIMD simulations.
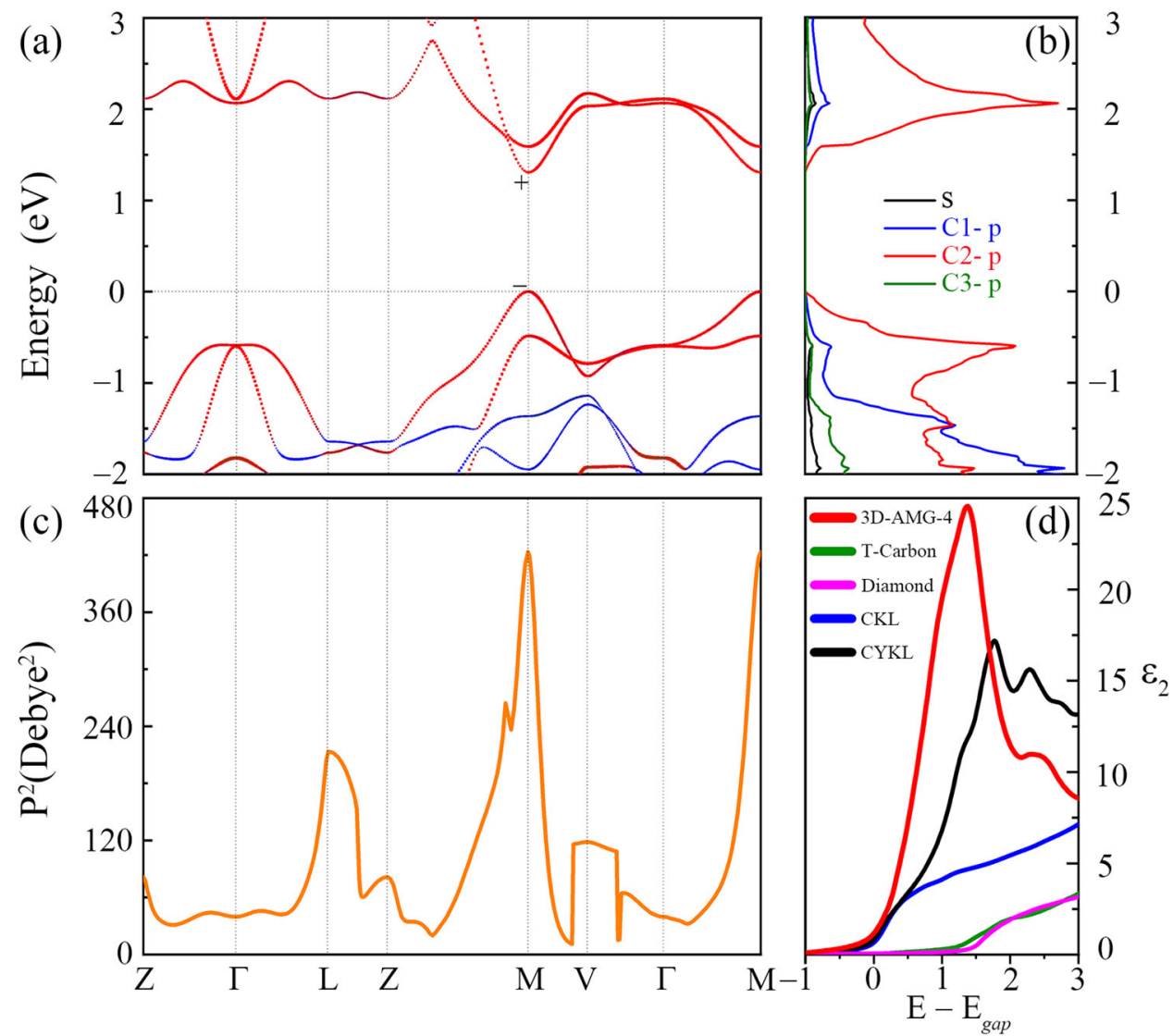

Fig. 3 Electronic and optical properties. a Band structure and b projected density of states (PDOS) of 3D-AMG-4. The band structure is colored with different contributions from $p$ orbitals of C1 atoms (blue), C2 atoms (red), and C3 atoms (green). c Transition matrix elements for 3D-AMG-4. d The imaginary part of the dielectric function of 3D-AMG-4 as a function of $E-E_{\text {gap }}$, where $E_{\text {gap }}$ is the band gap, in comparison with those of T-carbon, diamond, CKL, and CYKL under the HSE level. 
winding number $N=\frac{1}{\pi} \oint_{1} \operatorname{Tr}\left[\boldsymbol{A}_{\boldsymbol{k}}\right] \cdot d \boldsymbol{k}$ where $\boldsymbol{A}_{\boldsymbol{k}}$ is the Berry connection at point $\boldsymbol{k}$ for the occupied bands, and the integration path $I$ is around a loop encircling the nodal ring. This winding number is essentially the Berry phase in a unit of $\pi$. Our calculation confirms that $N=1$ for the nodal ring, indicating its topological character.

One significant consequence of topological nodal-ring semimetal is the presence of "drumhead-like" surface states. Here, we study the surface spectrum (Fig. 4c, d) of 3D-AMG-5 on the (001) surface. The result confirms that the "drumhead-like" surface states do exist and they are nestled outside the projected nodal ring in Fig. 4d (the red dashed lines). These "drumhead-like" surface states are slightly below the Fermi level; hence they should be easily detected by angle-resolved photoemission spectroscopy. For 3D-AMG-2 and 3D-AMG-8, also possess nodal rings, but the patterns of the nodal rings and surface states are different (see supplementary Fig. 8). In particular, 3D-AMG-2 has two helical nodal loops in the first BZ.

We would like to point out the electronic properties of 3D-AMGs are mainly contributed by the atoms in the armchair nanoribbons (i.e., the C2 atoms). This implies that the electronic properties of 3D-AMGs are intimately connected with those of armchair nanoribbons. As analyzed by Son et al. ${ }^{48}$, the band gaps of the armchair nanoribbons with a width equal to $3 p+2$ (where $p$ is a positive integer) are much smaller than those of the others, owing to the quantum confinement effect; Likewise, although local details of the armchair nanoribbons are perturbed by the inserted acetylenic linkages, the whole tendency of electronic properties of
3D-AMGs are partially consistent with those of the armchair nanoribbons. Specially speaking, 3D-AMG- $n$ with $n=3 p+2$ are semimetals, and the others are semiconductors.

\section{DISCUSSION}

Before closing, we would like to give some remarks about our work. The Shockley-Queisser limit ${ }^{47}$ suggests that the theoretically maximum solar converting efficiency $33.7 \%$ of a single-junction solar cell occurs for a semiconductor with a band gap of $1.34 \mathrm{eV}$ as evidenced by silicon ${ }^{49}, \mathrm{GaAs}^{50}, \mathrm{Culn}_{\mathrm{x}} \mathrm{Ga}_{1-\mathrm{x}} \mathrm{Se}_{2}{ }^{51}$, and hybrid organic-inorganic perovskite compounds $\left(\mathrm{CH}_{3} \mathrm{NH}_{3} \mathrm{PbX}_{3}, \mathrm{X}=\mathrm{I}, \mathrm{Br}\right.$, and $\mathrm{Cl})^{52}$. Nevertheless, these materials proposed so far have various limitations. For example, silicon is an indirect-band gap $(1.1 \mathrm{eV})$ semiconductor and its optical absorption is limited by the requirement of phonon assistance. $\mathrm{As}, \mathrm{Cd}$, and $\mathrm{Pb}$ are toxic; In is a

Table 2. The electron and hole effective masses as well as the PBE (HSE06) band gaps for CKL, CYKL, T-carbon, and 3D-AMG-4.

\begin{tabular}{lllllll}
\hline System & $m_{e}^{\|}$ & $\boldsymbol{m}_{\mathrm{lh}}^{\|}$ & $\boldsymbol{m}_{\mathrm{hh}}^{\|}$ & $\boldsymbol{m}_{e}^{\perp}$ & $\boldsymbol{m}_{h}^{\perp}$ & $E_{\text {gap }}(\mathrm{eV})$ \\
\hline CKL & 0.16 & -0.12 & -0.51 & 1.23 & -0.91 & $2.26(3.27)$ \\
CYKL & 0.34 & -0.33 & -1.09 & 0.31 & -0.61 & $1.81(2.71)$ \\
T-Carbon & 3.98 & -0.18 & -0.52 & 3.54 & -0.27 & $1.47(2.18)$ \\
3D-AMG-4 & 0.39 & -0.39 & -0.39 & 1.30 & -1.67 & $1.31(1.87)$ \\
\hline
\end{tabular}

The effective masses are given in units of the free electron mass.
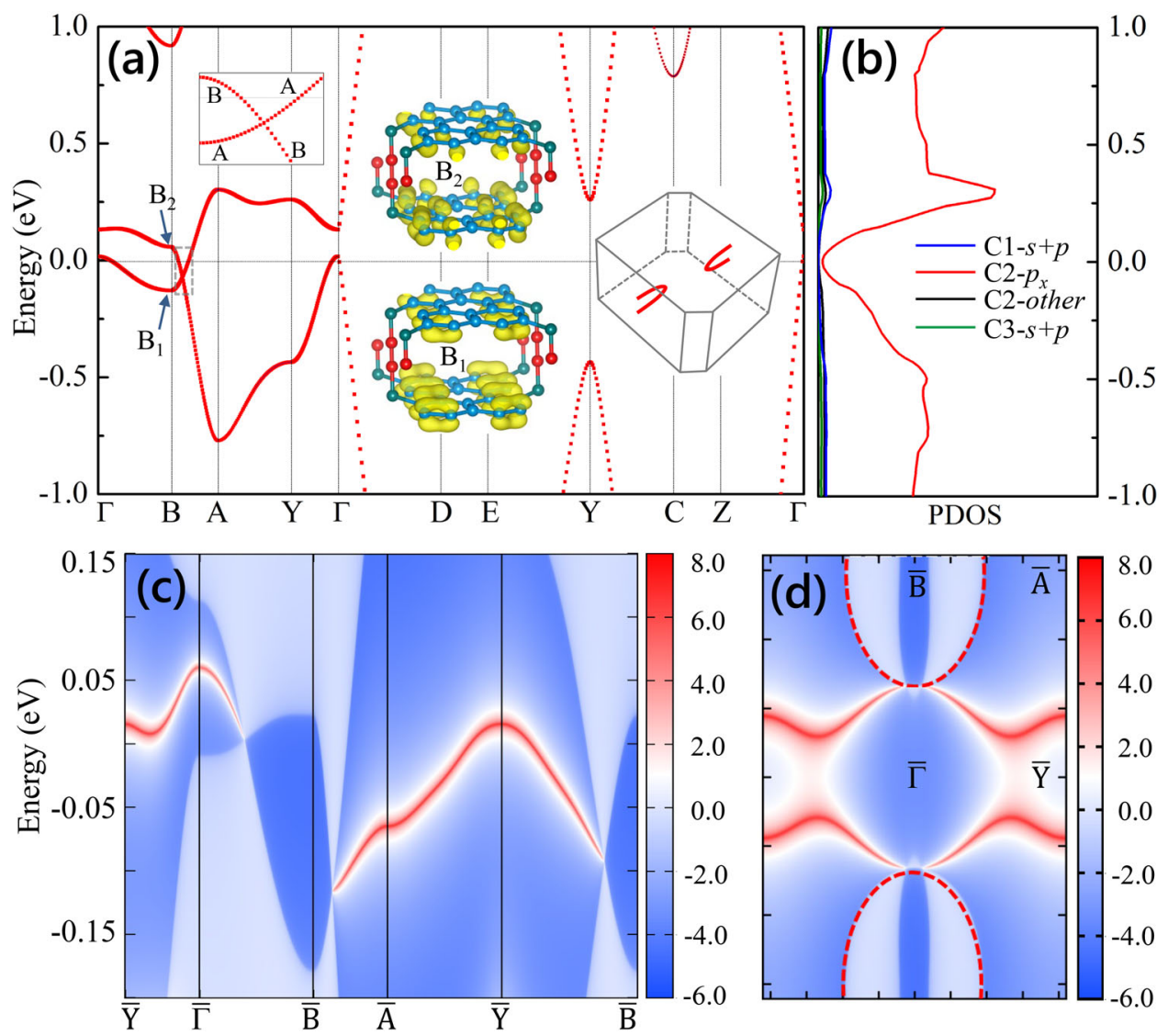

Fig. 4 Band structure of 3D-AMG-5 and its topological state. a Band structure of 3D-AMG-5, where the red bands correspond to the $p_{x}$ orbitals of $\mathrm{C} 2$ atoms (see the inset in Fig. 1b). The orbitals of other carbon atoms are insignificant around the Fermi level. Inset shows the side views of charge densities at $B_{1}$ and $B_{2}$ points and the perspective view of the topological nodal ring in the first $B Z$, respectively. $A$ and $B$ indicate the irreducible representations of the band crossing point along the B-A path. $\mathbf{b}$ The PDOS for 3D-AMG-5, in which the states around the Fermi level are mainly contributed by the $p_{x}$ orbital of $C 2$ atoms. c Surface energy bands and $\mathbf{d}$ surface spectrum at a fixed energy $E-E_{\mathrm{f}}=0$, where the red dashed lines show the projection of the red nodal ring on the (001) surface. 
rare element; and $\mathrm{CH}_{3} \mathrm{NH}_{3} \mathrm{PbX}_{3}$ is not stable ${ }^{53}$. In comparison with these well-known materials, the 3D-AMGs may be better candidates, since they are non-toxic, carbon is cheap and abundant, and most importantly, their band gaps are adjustable.

To guide the experiment, we simulate $\mathrm{X}$-ray diffraction peaks of 3D-AMGs and contrast them with those of representative carbon allotropes (see supplementary Fig. 10). Taking 3D-AMG-4 as an example, we also suggest a possible experimental scheme for its synthesis (see supplementary Fig. 11). One may start from the graphene, and functionalize the graphene with bromide ${ }^{54}$. Then, stacking the multilayer brominated graphene sheets and inserting acetylene molecules, and finally, deacidification will lead to the formation of 3D-AMG-4. Each step in this process is either already achieved or has a high chance to be achieved because of the existence of similar reactions. Given its energetic and dynamic stabilities and in view of the rapid progress in experimental techniques, which have realized a number of carbon structures in recent decades, we expect 3D-AMGs could also be realized in the near future.

Last but not least, it is worthily noted that 3D-AMGs would have several other advantages and promising applications. For instance, the large specific surface and space can enhance reversible storage space and storage capacity for lithium and sodium ions. Moreover, 3D-AMGs have the graphene-like basic unit, so they may inherit the excellent properties of graphene, such as superhigh conductivity and super-high thermal conductivity.

In this work, we propose a new strategy to tailor the properties of graphene through stitching different graphene layers with acetylenic linkages. According to the different connected ways, a new family of 3D carbon networks is obtained, namely 3D-AMG- $n$ here. 3D-AMGs show very excellent stability in that they are constructed from graphene. More strikingly, 3D-AMGs are not trivial carbon phases, actually they manifest many fascinating properties. For instance, 3D-AMG-3/4/7/10 are dipole-allowed truly direct band gap semiconductors with band gaps ranging from $1.07 \mathrm{eV}$ to $1.87 \mathrm{eV}$ and possess very outstanding optical absorption strength in comparison with other semiconducting carbon allotropes, which makes them quite suitable for optoelectronic or photovoltaic applications. On the other side, 3D-AMG-2/5/8 are topological semimetals, which is a hot topic in the study of topological materials. Moreover, 3D-AMGs have large specific surface and space, making them also preferable as catalysis, molecule sieves, and Li-ion anode materials, etc.

\section{METHODS}

\section{First-principles calculations}

The first-principles electronic structure calculations were based on the DFT with the Perdew-Burke-Ernzerhof (PBE) ${ }^{55}$ approximation to the exchangecorrelation energy. The core-valence interactions were described by the projector augmented wave (PAW) ${ }^{56}$ method, as implemented in the VASP package ${ }^{57,58}$. The kinetic energy cutoff of $550 \mathrm{eV}$ was adopted for the plane-wave basis. The atomic positions were fully relaxed by the conjugate gradient method; the energy and force convergence criteria were set to be $10^{-6} \mathrm{eV}$ and $10^{-3} \mathrm{eV} / \AA \AA$, respectively. The $k$-point meshes $7 \times 7 \times 7$ and $9 \times$ $7 \times 11$ were used for the BZ integration of 3D-AMG-4 and 3D-AMG-5, respectively. The band gaps of semiconductors were also calculated by using the Heyd-Scuseria-Ernzerhof (HSE06) hybrid functional ${ }^{59}$. The frequency-dependent dielectric matrix was calculated using the method described by Gajdos et al. within PAW potentials ${ }^{60}$. In order to study the dynamical stability, we used a finite displacement approach as implemented in the PHONOPY package ${ }^{61}$ to calculate the phonon dispersions. The thermal stability was investigated with the AIMD simulations in a canonical ensemble with a Nose-Hoover thermostat ${ }^{62,63}$. The band crossing characteristics were analyzed with the WannierTools ${ }^{64}$ package according to the TB model constructed via the Wannier90 code $^{65}$.

\section{DATA AVAILABILITY}

The data that support the findings of this study are available from the corresponding author upon reasonable request.

Received: 28 February 2021; Accepted: 16 June 2021; Published online: 16 July 2021

\section{REFERENCES}

1. Segawa, Y., Ito, H. \& Itami, K. Structurally uniform and atomically precise carbon nanostructures. Nat. Rev. Mater. 1, 15002 (2016).

2. Zhang, R.-S. \& Jiang, J.-W. The art of designing carbon allotropes. Front. Phys. 14, 13401 (2018).

3. Ajayan, P. M. The nano-revolution spawned by carbon. Nature $\mathbf{5 7 5}, \mathbf{4 9 - 5 0}$ (2019).

4. Gibbs, W. W. A new form of pure carbon dazzles and attracts. Science 366, 782-783 (2019).

5. Itami, K. \& Maekawa, T. Molecular nanocarbon science: present and future. Nano Lett. 20, 4718-4720 (2020).

6. Gao, Y. et al. Theoretical design of all-carbon networks with intrinsic magnetism. Carbon 177, 11-18 (2021).

7. Yue, Y. et al. Hierarchically structured diamond composite with exceptional toughness. Nature 582, 370-374 (2020).

8. Hirsch, A. The era of carbon allotropes. Nat. Mater. 9, 868-871 (2010).

9. Dresselhaus, M. S. On the past and present of carbon nanostructures. Phys. Status Solidi 248, 1566-1574 (2011).

10. Gao, X., Liu, H., Wang, D. \& Zhang, J. Graphdiyne: synthesis, properties, and applications. Chem. Soc. Rev. 48, 908-936 (2019).

11. Ma, Y. \& Chen, Y. Three-dimensional graphene networks: synthesis, properties and applications. Natl Sci. Rev. 2, 40-53 (2015).

12. Peurifoy, S. R. et al. Three-dimensional graphene nanostructures. J. Am. Chem. Soc. 140, 9341-9345 (2018).

13. Chen, W. et al. Polymeric graphene bulk materials with a $3 \mathrm{~d}$ cross-linked monolithic graphene network. Adv. Mater. 31, 1802403 (2019).

14. Zhao, K. et al. Super-elasticity of three-dimensionally cross-linked graphene materials all the way to deep cryogenic temperatures. Sci. Adv. 5, eaav2589 (2019).

15. Kroto, H. W., Heath, J. R., O'Brien, S. C., Curl, R. F. \& Smalley, R. E. C60: Buckminsterfullerene. Nature 318, 162-163 (1985).

16. lijima, S. Helical microtubules of graphitic carbon. Nature 354, 56-58 (1991).

17. De Volder, M. F., Tawfick, S. H., Baughman, R. H. \& Hart, A. J. Carbon nanotubes: present and future commercial applications. Science 339, 535-539 (2013).

18. Geim, A. K. \& Novoselov, K. S. The rise of graphene. Nat. Mater. 6, 183-191 (2007).

19. Georgakilas, V., Perman, J. A., Tucek, J. \& Zboril, R. Broad family of carbon nanoallotropes: classification, chemistry, and applications of fullerenes, carbon dots, nanotubes, graphene, nanodiamonds, and combined superstructures. Chem. Rev. 115, 4744-4822 (2015).

20. Liu, L. et al. Aligned, high-density semiconducting carbon nanotube arrays for high-performance electronics. Science 368, 850-856 (2020).

21. Avouris, P., Chen, Z. \& Perebeinos, V. Carbon-based electronics. Nat. Nanotechnol. 2, 605-615 (2007).

22. Yu, P. Y., Cardona, M. Fundamentals of semiconductors: physics and materials properties. (Springer, 2010).

23. Zhang, J. et al. Pseudo-topotactic conversion of carbon nanotubes to T-carbon nanowires under picosecond laser irradiation in methanol. Nat. Commun. 8, 683 (2017).

24. $\mathrm{Xu}, \mathrm{K}$. et al. Preparation of T-carbon by plasma enhanced chemical vapor deposition. Carbon 157, 270-276 (2020).

25. Chen, Y. et al. Carbon kagome lattice and orbital-frustration-induced metalinsulator transition for optoelectronics. Phys. Rev. Lett. 113, 085501 (2014).

26. Zhong, C., Xie, Y., Chen, Y. \& Zhang, S. Coexistence of flat bands and Dirac bands in a carbon-Kagome-lattice family. Carbon 99, 65-70 (2016).

27. Zhong, C., Zhang, W., Ding, G. \& He, J. Three-dimensional graphene networks modified with acetylenic linkages for high-performance optoelectronics and $\mathrm{Li}$ ion battery anode material. Carbon 154, 478-484 (2019).

28. Bansil, A., Lin, H. \& Das, T. Colloquium: topological band theory. Rev. Mod. Phys. 88, 021004 (2016).

29. Liu, P., Williams, J. R. \& Cha, J. J. Topological nanomaterials. Nat. Rev. Mater. 4, 479-496 (2019).

30. Giustino, F. et al. The 2021 quantum materials roadmap. J. Phys. Mater 3, 4 (2021).

31. Zhang, Y., Tan, Y. W., Stormer, H. L. \& Kim, P. Experimental observation of the quantum Hall effect and Berry's phase in graphene. Nature 438, 201-204 (2005). 
32. Schnyder, A. P., Ryu, S., Furusaki, A. \& Ludwig, A. W. W. Classification of topological insulators and superconductors in three spatial dimensions. Phys. Rev. B 78, 195125 (2008).

33. Zhong, C. et al. Two-dimensional honeycomb borophene oxide: strong anisotropy and nodal loop transformation. Nanoscale 11, 2468-2475 (2019).

34. Gao, Y. et al. Hexagonal supertetrahedral boron: a topological metal with multiple spin-orbit-free emergent fermions. Phys. Rev. Mater. 3, 044202 (2019).

35. Zhou, X.-F. et al. Semimetallic two-dimensional boron allotrope with massless dirac fermions. Phys. Rev. Lett. 112, 085502 (2014).

36. He, X.-L. et al. Predicting three-dimensional icosahedron-based boron B60. Phys. Rev. B 99, 184111 (2019).

37. Weng, $H$. et al. Topological node-line semimetal in three-dimensional graphene networks. Phys. Rev. B 92, 045108 (2015).

38. Wang, J. T. et al. Body-centered orthorhombic C16: a novel topological node-line semimetal. Phys. Rev. Lett. 116, 195501 (2016).

39. Gao, Y. et al. A class of topological nodal rings and its realization in carbon networks. Phys. Rev. B 97, 121108 (2018).

40. Zhong, $C$. et al. Towards three-dimensional Weyl-surface semimetals in graphene networks. Nanoscale 8, 7232-7239 (2016).

41. Wu, W. et al. Nodal surface semimetals: theory and material realization. Phys. Rev. B 97, 115125 (2018).

42. Chen, S. Z., Li, S., Chen, Y. \& Duan, W. Nodal flexible-surface semimetals: case of carbon nanotube networks. Nano Lett. 20, 5400-5407 (2020).

43. Zhong, C. et al. Three-dimensional Pentagon Carbon with a genesis of emergent fermions. Nat. Commun. 8, 15641 (2017).

44. $\mathrm{Hu}$, J. et al. Three-dimensional honeycomb carbon: Junction line distortion and novel emergent fermions. Carbon 141, 417-426 (2019).

45. Singh, V. et al. Graphene based materials: past, present and future. Prog. Mater. Sci. 56, 1178-1271 (2011).

46. Meng, W. et al. Parity-forbidden transitions and their impact on the optical absorption properties of lead-free metal halide perovskites and double perovskites. J. Phys. Chem. Lett. 8, 2999-3007 (2017).

47. Shockley, W. \& Queisser, H. J. Detailed balance limit of efficiency of p-n junction solar cells. J. Appl. Phys. 32, 510-519 (1961).

48. Son, Y.-W., Cohen, M. L. \& Louie, S. G. Energy gaps in graphene nanoribbons. Phys. Rev. Lett. 97, 216803 (2006).

49. Wang, Q. et al. Direct band gap silicon allotropes. J. Am. Chem. Soc. 136, 9826-9829 (2014).

50. Kosten, E. D., Atwater, J. H., Parsons, J., Polman, A. \& Atwater, H. A. Highly efficient GaAs solar cells by limiting light emission angle. Light Sci. Appl. 2, e45-e45 (2013).

51. Gabor, A. M. et al. High-efficiency CulnxGa1-xSe2solar cells made from (Inx,Ga1 -x)2Se3precursor films. Appl. Phys. Lett. 65, 198-200 (1994).

52. Liu, M., Johnston, M. B. \& Snaith, H. J. Efficient planar heterojunction perovskite solar cells by vapour deposition. Nature 501, 395-398 (2013).

53. Polman, A., Knight, M., Garnett, E. C., Ehrler, B. \& Sinke, W. C. Photovoltaic materials: present efficiencies and future challenges. Science 352, aad4424 (2016).

54. Jankovsky, O. et al. Towards graphene bromide: bromination of graphite oxide. Nanoscale 6, 6065-6074 (2014).

55. Perdew, J. P. et al. Atoms, molecules, solids, and surfaces: applications of the generalized gradient approximation for exchange and correlation. Phys. Rev. B $\mathbf{4 6}$ 6671-6687 (1992).

56. Blöchl, P. E. Projector augmented-wave method. Phys. Rev. B 50, 17953-17979 (1994)

57. Kresse, G. \& Furthmüller, J. Efficient iterative schemes for ab initio total-energy calculations using a plane-wave basis set. Phys. Rev. B 54, 11169-11186 (1996).

58. Kresse, G. \& Joubert, D. From ultrasoft pseudopotentials to the projector augmented-wave method. Phys. Rev. B 59, 1758-1775 (1999).

59. Heyd, J., Scuseria, G. E. \& Ernzerhof, M. Hybrid functionals based on a screened Coulomb potential. J. Chem. Phys. 118, 8207-8215 (2003).

60. Gajdoš, M., Hummer, K., Kresse, G.,Furthmüller, J. \& Bechstedt, F. Linear optical properties in the projector-augmented wave methodology. Phys. Rev. B 73 045112 (2006).

61. Togo, A., Oba, F. \& Tanaka, I. First-principles calculations of the ferroelastic transition between rutile-type and $\mathrm{CaCl} 2$-type $\mathrm{SiO} 2$ at high pressures. Phys. Rev. $B$ 78, 134106 (2008).

62. Evans, D. J. \& Holian, B. L. The Nose-Hoover thermostat. J. Chem. Phys. 83, 4069-4074 (1985).
63. Nosé, S. A unified formulation of the constant temperature molecular dynamics methods. J. Chem. Phys. 81, 511-519 (1984).

64. Wu, Q., Zhang, S., Song, H.-F., Troyer, M. \& Soluyanov, A. A. WannierTools: an open-source software package for novel topological materials. Comput. Phys. Commun. 224, 405-416 (2018).

65. Pizzi, G. et al. Wannier90 as a community code: new features and applications. J. Phys. Condens. Matter 32, 165902 (2020).

66. Li, Z.-Z., Wang, J.-T., Mizuseki, H. \& Chen, C. Computational discovery of a new rhombohedral diamond phase. Phys. Rev. B 98, 094107 (2018).

67. Occelli, F., Loubeyre, P. \& LeToullec, R. Properties of diamond under hydrostatic pressures up to $140 \mathrm{GPa}$. Nat. Mater. 2, 151-154 (2003).

68. Sun, P.-P., Bai, L., Kripalani, D. R. \& Zhou, K. A new carbon phase with direct bandgap and high carrier mobility as electron transport material for perovskite solar cells. npj. Comput. Mater. 5, 9 (2019).

\section{ACKNOWLEDGEMENTS}

We wish to thank Peng-Jie Guo, Zhongwei Zhang, and Weikang Wu for helpful discussions. This work was supported by the National Key R\&D Program of China (Grants no. 2019YFA0308603 and 2017YFA0302903), the National Natural Science Foundation of China (Grants no. 11934020, 11774424, and 11804039), and the Singapore Ministry of Education AcRF Tier 2 (MOE2017-T2-2-108). Y.G. was supported by the Outstanding Innovative Talents Cultivation Funded Programs 2021 of Renmin University of China. Computational resources were provided by the Physical Laboratory of High-Performance Computing at the Renmin University of China.

\section{AUTHOR CONTRIBUTIONS}

Y.G. and C.Z. contributed equally to this work. Y.G. and C.Z., investigation, data curation, and Writing-original draft preparation, Methodology, Software, and Writingreviewing and editing. S.A.Y., writing-reviewing, and editing. K.L. and Z.-Y.L. conceptualization, supervision, and project administration.

\section{COMPETING INTERESTS}

The authors declare no competing interests.

\section{ADDITIONAL INFORMATION}

Supplementary information The online version contains supplementary material available at https://doi.org/10.1038/s41524-021-00579-5.

Correspondence and requests for materials should be addressed to K.L. or Z.-Y.L.

Reprints and permission information is available at http://www.nature.com/ reprints

Publisher's note Springer Nature remains neutral with regard to jurisdictional claims in published maps and institutional affiliations.

Open Access This article is licensed under a Creative Commons
Attribution 4.0 International License, which permits use, sharing,
adaptation, distribution and reproduction in any medium or format, as long as you give
appropriate credit to the original author(s) and the source, provide a link to the Creative
Commons license, and indicate if changes were made. The images or other third party
material in this article are included in the article's Creative Commons license, unless
indicated otherwise in a credit line to the material. If material is not included in the
article's Creative Commons license and your intended use is not permitted by statutory
regulation or exceeds the permitted use, you will need to obtain permission directly
from the copyright holder. To view a copy of this license, visit http://creativecommons.
org/licenses/by/4.0\%

(c) The Author(s) 2021 